\title{
Restoring symmetry in two-dimensional solid-state NMR correlation spectra is
}

\author{
Jose-Enrique Herbert-Pucheta, Philippe Pelupessy, Geoffrey Bodenhausen, Piotr Tekely*
}

Ecole Normale Supérieure, Département de Chimie, 24 rue Lhomond, 75231 Paris cedex 05, France

Université Pierre-et-Marie Curie, Paris, France

UMR 7203 Laboratoire des Biomolécules, CNRS/UPMC/ENS, Paris, France

\section{A R T I C L E I N F O}

\section{Article history:}

Received 29 March 2012

In final form 14 May 2012

Available online 23 May 2012

\begin{abstract}
A B S T R A C T
The intrinsic asymmetry of 2D solid-state homonuclear NMR correlation spectra that arises from a nonuniform preparation of the magnetization can be removed by equilibrating the magnetization in the initial stage of the experiments through dipolar recoupling induced by PARIS or PARISxy schemes. Both methods ensure magnetization exchange with modest radio-frequency amplitudes and permit one to create an initial state that restores the symmetry about the main diagonal of 2D spectra of uniformly labeled biomolecules. This improves the determination of structural and kinetic information. Both recoupling schemes may also be used to record nearly quantitative peak amplitudes in 1D cross-polarization magic-angle-spinning spectra.
\end{abstract}

(c) 2012 Elsevier B.V. All rights reserved.

\section{Introduction}

Two-dimensional (2D) homonuclear correlation spectroscopy provides a powerful tool for the identification of spectral connectivities, the assignment of resonance lines, and the determination of internuclear distances and exchange rates [1]. Efficient magnetization transfer between spins $S$ such as carbon-13 or nitrogen-15 is a prerequisite for the assignment of solid-state NMR spectra of isotopically enriched molecules. Magnetization transfer can be brought about either via through-bond scalar couplings or via through-space dipolar interactions, provided the latter are 'recoupled' to cancel their elimination by magic angle spinning (MAS). To promote dipolar recoupling, we developed PARIS [2] and PARISxy [3] methods which permit one to achieve an efficient exchange of magnetization over a wide range of spinning frequencies and static magnetic fields and to record sensitive 2D correlation spectra of microcrystalline proteins [4], amyloid fibrils [5] and mixtures of crystallographic forms [6].

In solids, ${ }^{13} \mathrm{C}-{ }^{13} \mathrm{C}$ correlation spectra can be inherently asymmetric when cross-polarization from protons to carbons is employed before the evolution period, or, if the relaxation rates are not equal, when the delay between successive acquisitions is too short to allow a uniform recovery of the longitudinal magnetization. In spectra recorded by cross polarization, the asymmetry is most severe for cross-peaks between proton-carrying carbons on

First presented at the 53rd Rocky Mountain Conference on Analytical Chemistry, August 2010, Snowmass, Colorado, USA.

* Corresponding author at: Ecole Normale Supérieure, Département de Chimie, 24 rue Lhomond, 75231 Paris cedex 05, France.

E-mail address: Piotr.Tekely@ens.fr (P. Tekely). the one hand, and carbonyl, carboxyl, or quaternary carbons on the other, since the latters' magnetization suffers from a slow build-up during cross-polarization. Asymmetric cross-peak intensities can also arise because of local variations in internal mobility. Whatever their origin, the asymmetry of $2 \mathrm{D}{ }^{13} \mathrm{C}-{ }^{13} \mathrm{C}$ correlation spectra tend to complicate the assignment of uniformly labeled biomolecules and hamper the extraction of reliable structural and kinetic parameters. In order to record symmetric 2D spectra, it is desirable to equilibrate the magnetization in the initial stages of the experiments, preferably on a short time scale to avoid losses due to relaxation. It has been shown that the symmetry of the aliphatic region of a $2 \mathrm{D}{ }^{13} \mathrm{C}-{ }^{13} \mathrm{C}$ correlation spectrum of ubiquitin could be improved by using rotary resonance based dipolar recoupling (RAD) [7] during an equilibration time [8] after crosspolarization. Taking advantage of efficient dipolar recoupling that can be promoted over wide spectral ranges by PARIS or PARISxy sequences, we present the first fully symmetric $2 \mathrm{D}{ }^{13} \mathrm{C}-{ }^{13} \mathrm{C}$ correlation spectra over the whole spectral range. We also show that the requirements that must be fulfilled to record symmetric correlation spectra are similar, although not identical, to the requirements to obtain quantitative CP-MAS spectra where the integrated signal intensities are proportional to the number of nuclei.

\section{Methods and materials}

All experiments were performed in a magnetic field of $9.4 \mathrm{~T}$ $\left(400 \mathrm{MHz}\right.$ for ${ }^{1} \mathrm{H}$ ) at spinning frequencies $v_{\text {rot }}$ between 20 and $30 \mathrm{kHz}$. The pulse sequences used in this work are shown in Figure 1. Either the phase-alternated recoupling irradiation scheme (PARIS) [2] or its phase-shifted counterpart PARISxy [3] were used 
(a)

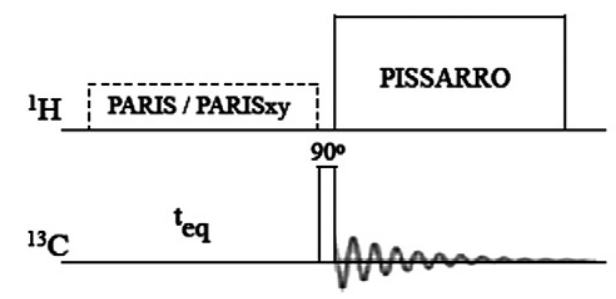

(b)
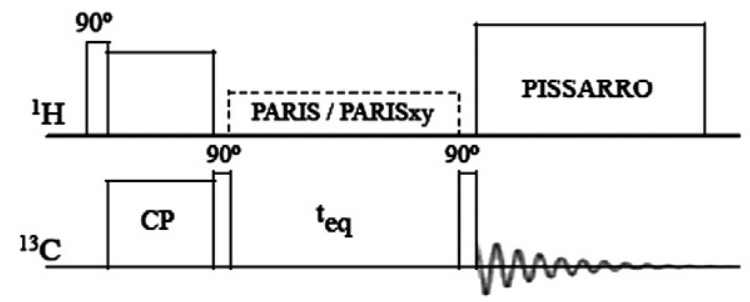

(c)
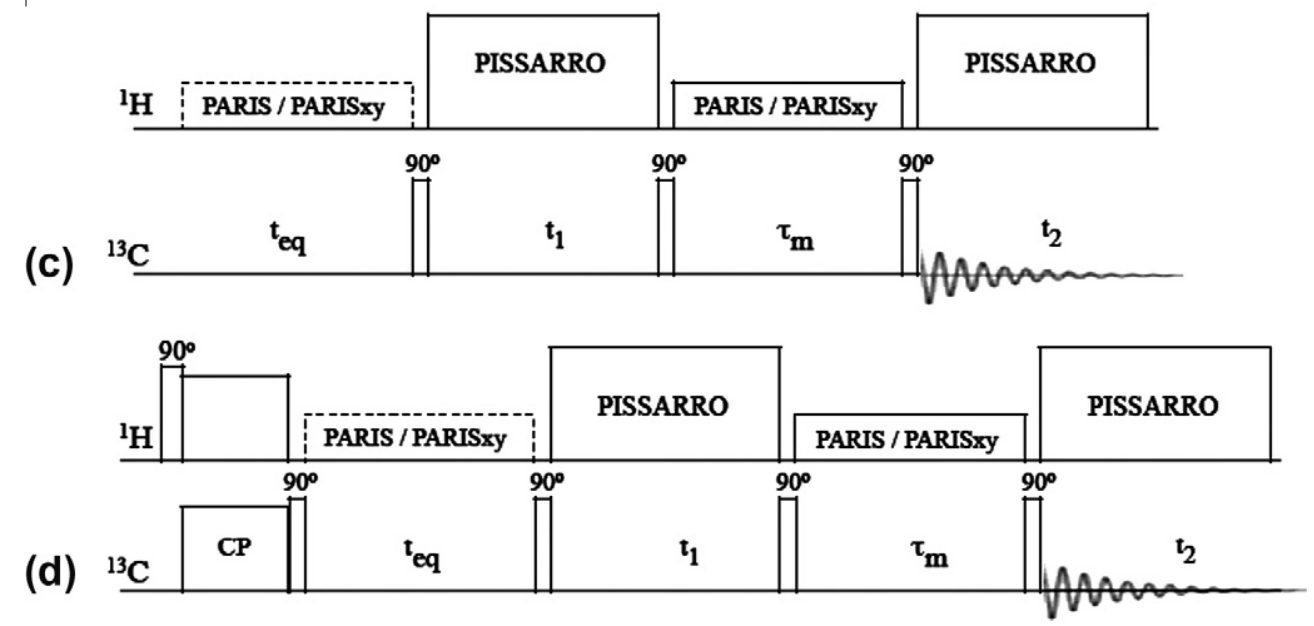

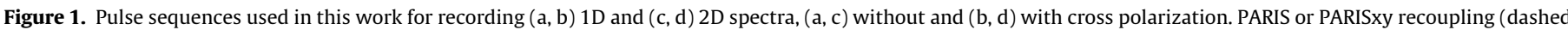

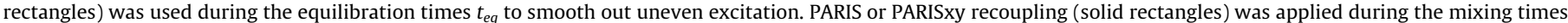
$\tau_{\mathrm{m}}$ to promote uniform exchange.

to equilibrate the magnetization (dashed rectangles in Figure 1). Both recoupling methods use $r f$ fields applied only to the protons with a moderate constant $r f$ amplitude $v_{1}\left({ }^{1} \mathrm{H}\right)$ that need not fulfill any rotary resonance condition [2,3]. For PARIS irradiation the phase is reversed from $+x$ to $-x$ after a duration $\tau_{\mathrm{p}}=N \tau_{\text {rot }}$ where $N=1 / 2$ or 2 . In this work we used only $N=2$, since the magnetization exchange between spectrally distant carbons such as carboxyl and aliphatic carbons (separated by ca. $100-150 \mathrm{ppm}$ or $10-15 \mathrm{kHz}$ at 9.4 T) is less efficient when using PARIS with $N=1 / 2$ [2], while PARIS with $N=2$ generates recoupling bands at $v_{\text {rot }} / 2$ on either side of resonance signals [2]. For PARISxy, the pulse duration $\tau_{\mathrm{p}}$ must always be equal to half the rotor period $\left(\tau_{\mathrm{p}}=\tau_{\text {rot }} / 2\right)$. PARISxy consists of a block of $m$ pairs ( $m=1$ or 2 ) of phase-alternated pulses $[(x)(-x)]_{m}$, followed by a phase-shifted block $[(y)(-y)]_{m}[3]$. Each version creates different sets of recoupling bands which allows their complementary use and flexible exploitation over a very broad range of spinning frequencies and static magnetic fields [3-6]. In the present work, with moderate spinning frequencies $20<v_{\text {rot }}<30 \mathrm{kHz}$ and a medium static magnetic field of $9.4 \mathrm{~T}$, PARISxy $(m=1)$ naturally appears to be the method of choice [3]. Heteronuclear PISSARRO decoupling [9-11] with an $r f$ amplitude $v_{1}\left({ }^{1} \mathrm{H}\right)=100 \mathrm{kHz}$ and a pulse duration $\tau_{\mathrm{p}}=0.9 \tau_{\text {rot }}$ was used during the evolution and acquisition periods. Polycrystalline samples of uniformly ${ }^{13} \mathrm{C},{ }^{15} \mathrm{~N}$-labeled L-alanine, L-arginine hydrochloride and L-histidine were purchased from different sources and used without further purification.

\section{Results and discussion}

Figure 2 illustrates the effect of the initial equilibration of magnetization on 1D and 2D spectra of L-histidine recorded with the pulse sequences of Figure $1 \mathrm{a}$ and $\mathrm{c}$. In the 1D spectra, the changes in peak intensities that result from equilibration show how the initial ${ }^{13} \mathrm{C}$ magnetization is redistributed within the spin system. This allows one to restore the symmetry about the main diagonal of $2 \mathrm{D}$ spectra (Figure 2 top right). Figure 3 shows that the recovered symmetry does not depend on the mixing time. The asymmetry parameters $\eta_{i j}$ are defined as ratios of peak volumes (integrated amplitudes) $\eta_{i j}=I_{i j} / I_{j i}$ for cross-peaks on either side of the diagonal that connect two chemically inequivalent sites $i$ and $j$. When the ratio $\eta_{i j}=1$, this is synonymous of full symmetry. We noticed that a similar improvement of the symmetry about the main diagonal can be also achieved at higher spinning frequencies, provided the equilibration time is longer (data not shown).

The intrinsic asymmetry of ${ }^{13} \mathrm{C}-{ }^{13} \mathrm{C}$ exchange spectra hampers the extraction of reliable kinetic parameters. This is visualized in Figure 4, which shows cross- and diagonal-peak volumes in proton-driven spin diffusion (PDSD) and PARISxy exchange spectra recorded without equilibration. The time-dependence of the crossand diagonal-peaks was fitted by assuming that the initial part of the build-up curves is governed by spin exchange between only two sites, which allows one to express the intensities of the diagonal peaks $I_{i i}$ and cross-peaks $I_{i j}$ as 

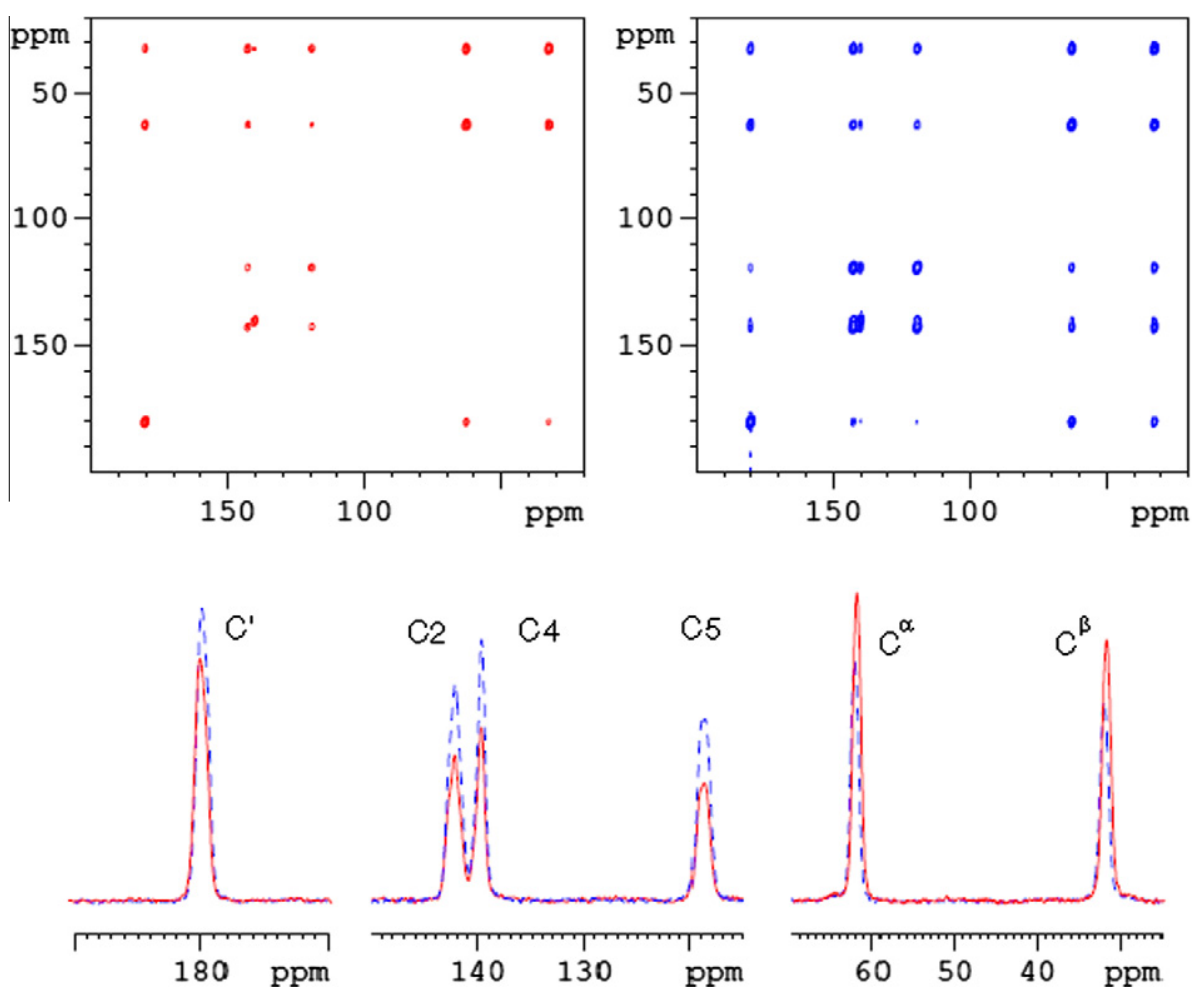

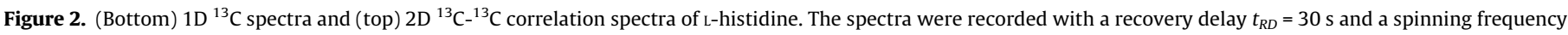

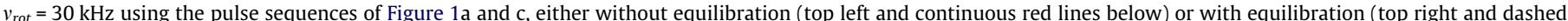

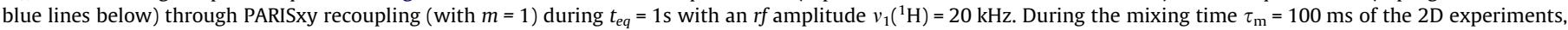

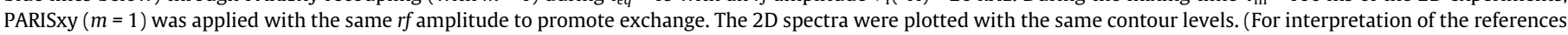
to colour in this figure legend, the reader is referred to the web version of this article.)

$I_{i i}=A_{i i} \exp \left(-R_{i i} \tau_{\mathrm{m}}\right)\left(1+\exp \left(-2 k_{i j} \tau_{\mathrm{m}}\right)\right)$

$I_{i j}=A_{i j} \exp \left(-R_{i j} \tau_{\mathrm{m}}\right)\left(1-\exp \left(-2 k_{i j} \tau_{\mathrm{m}}\right)\right)$

where $\tau_{\mathrm{m}}$ is the mixing time, while $k_{i j}$ is the exchange rate constant between two sites. The spin-lattice rate constants $R_{i i}$ describe the decay of the diagonal peak volumes, while the rate constants $R_{i j}$ describe the decaying envelopes of the cross-peaks. The differences between the fitted values of symmetry-related rate constants (the $k_{i j}$ resulting from fitting are unlike $k_{j i}$ ), and between sites with similar internuclear distances $r_{i j}$ and $r_{j k}\left(k_{i j}\right.$ are unlike $\left.k_{j k}\right)$, witness the difficulties of extracting reliable structural information from asymmetric exchange spectra. This stands in contrast to exchange data recorded after initial equilibration which, as shown in Figure 5, permits one not only to recover the symmetry about the main diagonal of the $2 \mathrm{D}$ spectra, but also to extract reliable exchange rate constants that correctly reflect the symmetry of the kinetic rate matrix.

Structural information about internuclear distances is available from spin exchange rate constants governing the polarization transfer (also called flip-flop or spin-diffusion processes) [12-15]. The exchange rate constant between spins $S_{i}$ and $S_{j}$ that are coupled to ${ }^{1} \mathrm{H}$ spins is given by [12]

$W_{i j}=\left(\frac{\pi}{2}\right) d_{i j}^{2} F_{i j}(\omega)$

where $d_{i j}$ denotes the effective dipolar coupling

$d_{i j}=-\left(\frac{\mu_{0}}{4 \pi}\right)\left(\frac{\gamma^{2} h}{r_{i j}^{3}}\right) \frac{1}{2}\left(3 \cos ^{2} \Theta_{i j}-1\right)$

and $r_{i j}$ is the internuclear distance, with the vector $\boldsymbol{r}_{i j}$ forming an angle $\Theta_{i j}$ with the external magnetic field. The term $F_{i j}(\omega)$ is the intensity of the normalized zero-quantum (ZQ) spectrum (flip-flop transition of the two spins $S_{i}$ and $\left.S_{j}\right)$ at zero frequency $(\omega=0)$ The shape of $F_{i j}(\omega)$ depends on the dipolar interactions and is centered on the difference of the resonances of the two spins. In static solids, the dependence of $W_{i j}$ on the spectral separation between the two spins via the $F_{i j}(\omega)$ term constitutes a complicating factor in interpreting spin-exchange rate constants in terms of internuclear distances. In spinning samples, this complication is alleviated as the dependence of the exchange rates on the chemical-shift difference via the $F_{i j}(\omega)$ term is significantly reduced by the averaging of the polarization transfer rate over a rotor cycle [13]. Assuming a weak collision approximation [14], the term $F_{i j}(\omega)$ is given by a zeroquantum relaxation time constant $\bar{T}_{2}^{(Z Q)}$ so that the powder average of the exchange rate constant under MAS may be written as

$\bar{W}_{i j}=\left(\frac{\pi}{2}\right) \bar{d}_{i j}^{2} \bar{T}_{2}^{(Z Q)}$

The zero-quantum relaxation rate is usually assumed to be approximately equal to the sum of the single-quantum relaxation rates of the two spins [15]. An estimate of $\bar{T}_{2}^{(Z Q)}$ to be used in Eq. (5) was obtained from the $1 / T_{2}$ rates of the corresponding ${ }^{13} \mathrm{C} \mathrm{nu}-$ clei, as measured in the presence of PARISxy irradiation during the mixing time. The factor $\left.\overline{P_{2}\left(\cos \Theta_{i j}\right.}\right)^{2}$ was taken to be equal to 0.25 [13]. The exchange rate constants $\overline{W_{i j}}$ calculated from Eq. (5) are given in Table 1 . The remarkable agreement between these calculated rate constants and those extracted by fitting the experimental data of Figure 5 to Eqs. (1), (2), although it may be perhaps somewhat fortuitous considering the number of assumptions and approximations, suggests that rate constants retrieved from symmetric $2 \mathrm{D}$ exchange spectra are directly related to internuclear distances and may be exploited for structure determination. This is analogous to the measurement of internuclear distances between chemically equivalent sites in rotating solids [16,17].

In experiments involving 3-site exchange, the time dependence of the longitudinal magnetization during the mixing time can be 
(b)
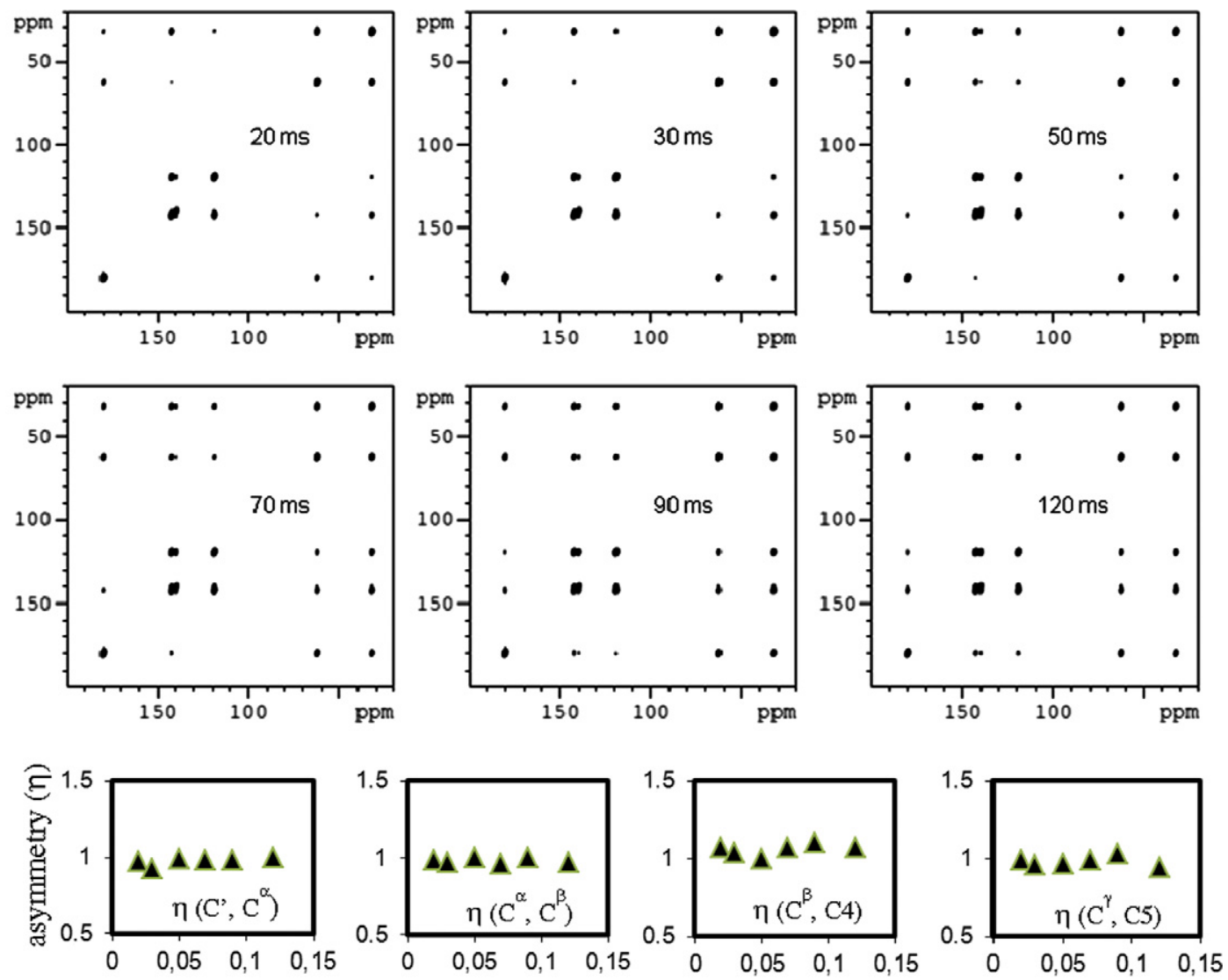

mixing time (s)

Figure 3. (a) Asymmetry parameters $\eta\left(\mathrm{C}^{\mathrm{i}}, \mathrm{C}^{\mathrm{j}}\right)$ and $(\mathrm{b}){ }^{13} \mathrm{C}-{ }^{13} \mathrm{C}$ exchange spectra of L-histidine as a function of the mixing time $\tau_{\mathrm{m}}$ using the sequence of Figure $1 \mathrm{c}$ with equilibration through PARISxy recoupling $(m=1)$ during a time $t_{e q}=1 \mathrm{~s}$ with an $r f$ amplitude $v_{1}\left({ }^{1} \mathrm{H}\right)=20 \mathrm{kHz}$. The experimental conditions were the same as those of Figure 2 .

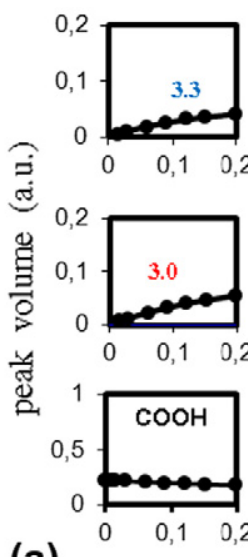

(a)

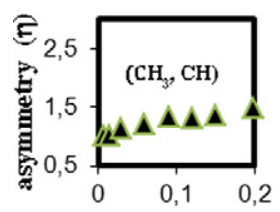

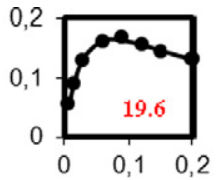
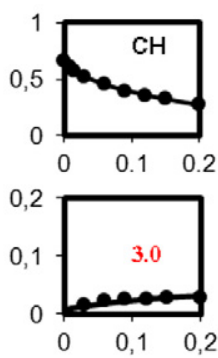

mixing time $(s)$
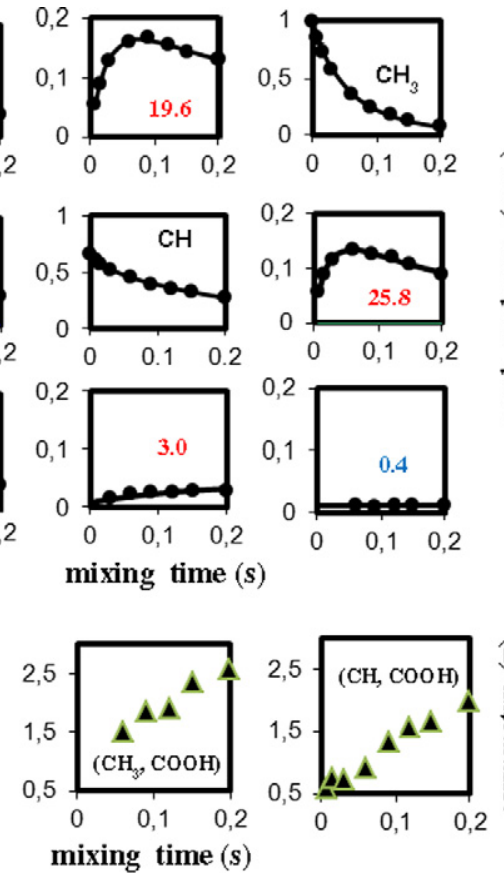
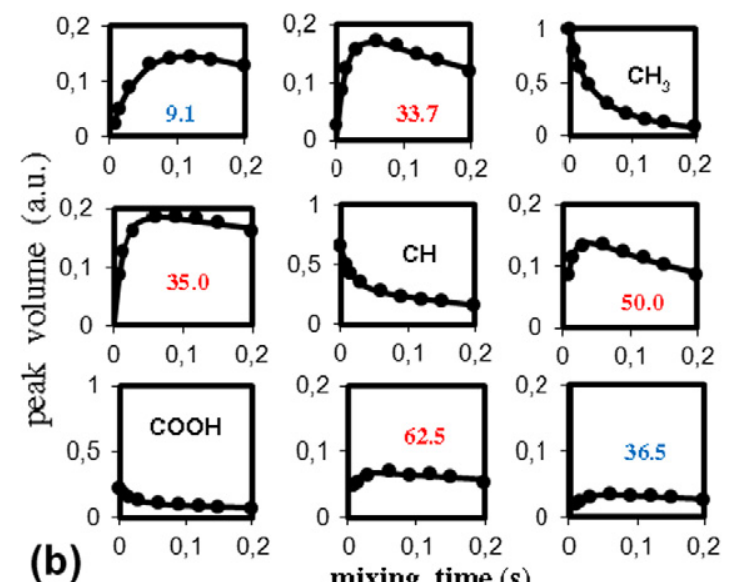

mixing time $(s)$
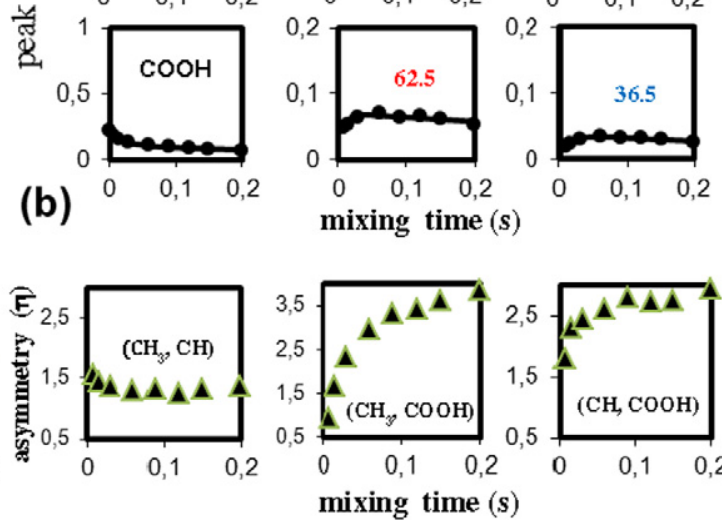

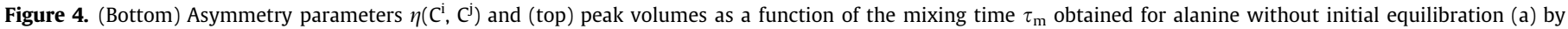

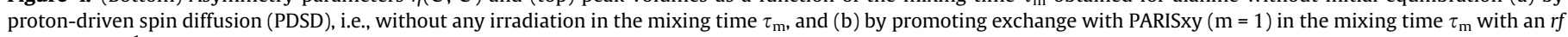

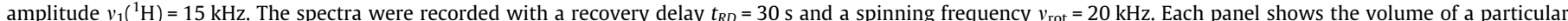

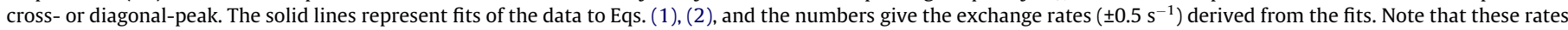
often violate the expected symmetry about the diagonal. 

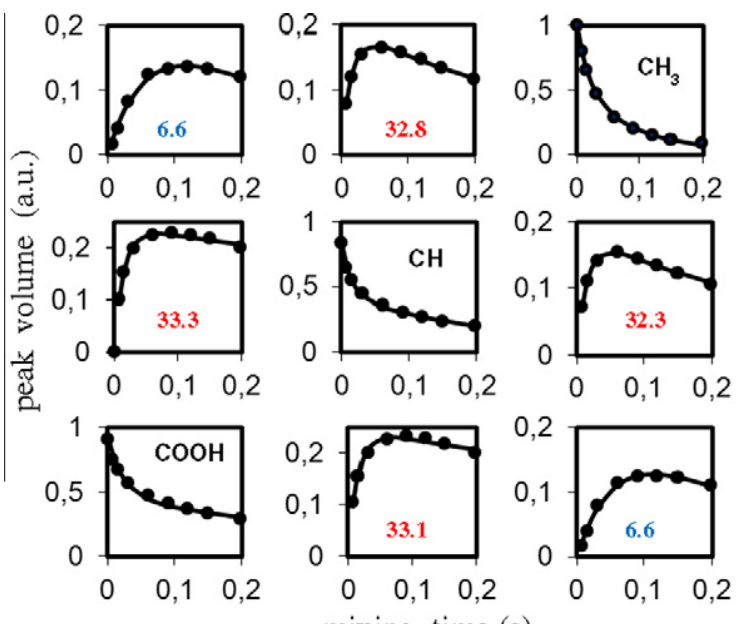

mixing time $(\mathrm{s})$
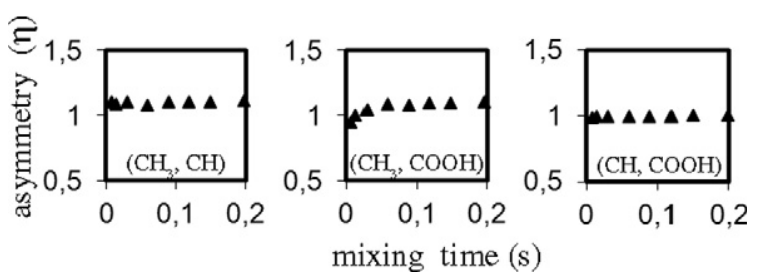

Figure 5. (Bottom) Asymmetry parameters $\eta\left(\mathrm{C}^{\mathrm{i}}, \mathrm{C}^{\mathrm{j}}\right)$ and (top) peak volumes as a function of the mixing time $\tau_{\mathrm{m}}$ obtained for alanine in analogy to Figure 4 but with initial equilibration using PARISxy $(\mathrm{m}=1)$ with an $r f$ amplitude $v_{1}\left({ }^{1} \mathrm{H}\right)=15 \mathrm{kHz}$ during $t_{e q}=1 \mathrm{~s}$, and by promoting exchange with PARISxy $(\mathrm{m}=1)$ in the mixing time $\tau_{\mathrm{m}}$ with the same $r f$ amplitude. The spectra were recorded with a recovery delay $t_{R D}=30 \mathrm{~s}$ and a spinning frequency $v_{\text {rot }}=20 \mathrm{kHz}$. Each panel shows the volume of a particular cross- or diagonal-peak. The solid lines represent fits of the data to Eqs. (1), (2), and the numbers give the exchange rates $\left( \pm 0.5 \mathrm{~s}^{-1}\right)$ derived from the fits (also see Table 1). Note that these rates fulfill the expected symmetry about the diagonal.

described more accurately by a system of coupled differential equations involving a matrix $L$ :

$L=\left(\begin{array}{ccc}-R_{1}-k_{12}-k_{13} & k_{12} & k_{13} \\ k_{21} & -R_{2}-k_{21}-k_{23} & k_{23} \\ k_{31} & k_{32} & -R_{3}-k_{31}-k_{32}\end{array}\right)$

However, as shown in Figure 6 for alanine, when assuming a single population in each site, we observed significant differences between the experimental and fitted build-up curves. Similar discrepancies show up in early PDSD [8] and dipolar assisted rotational resonance (DARR) [18] exchange spectra of alanine recorded without equilibration of the magnetization. The same problem appears in asymmetric 2D PARISxy exchange spectra obtained without equilibration (data not shown). This must be due to a non-uniform overlap of resonance lines that may be passively broadened (in PDSD) or actively broadened (in the presence of $r f$ irradiation) [2,18]. This non-uniform spectral overlap creates in turn two distinct spin populations endowed with a different efficiency of polarization exchange. Assuming an initial equilibration of magnetization, the time dependence can be described by a symmetric $6 \times 6$ exchange matrix that represents three sites $\{1,2,3\}$ and three sites $\left\{1^{\prime}, 2^{\prime}, 3^{\prime}\right\}$ that have pairwise identical chemical shifts but different exchange rates and unequal populations $p$ and $p^{\prime}$.

If $p=0.63$ and $p^{\prime}=0.37$, this description leads to satisfactory fits of the experimental data (solid blue lines in Figure 6). Finally, we were not able to obtain a good agreement when assuming the presence of two populations allowing for intermolecular exchange between chemically equivalent sites but without any exchange within the second population [18].

Highly symmetric 2D correlation or exchange spectra can also be recorded by equilibrating the initial magnetization after crosspolarization. This is shown in Figure 7 for alanine spectra obtained with the sequence of Figure 1d. As mentioned above, in spectra recorded with cross polarization, the asymmetry is most severe for cross-peaks between proton-carrying carbons on the one hand, and carbonyl, carboxyl, or quaternary carbons on the other. With a short cross-polarization contact time $t_{C P}=100 \mu \mathrm{s}$ followed by equilibration during $t_{e q}=1 \mathrm{~ms}$, this shows up dramatically through a strongly asymmetric spectrum (Figure 7 left). Yet, the symmetry can be fully restored under the same experimental conditions after equilibration during $t_{e q}=100 \mathrm{~ms}$ (Figure 7 right).

Finally it is worth pointing out that the requirements to record symmetric correlation or exchange spectra are similar to, although not exactly the same as, the requirements to obtain fully quantitative CP-MAS spectra in which the integrated intensities are proportional to the number of nuclei. Indeed, even without reaching a fully equilibrated initial state, the presence of populations with efficiently exchanging sites, which under PARIS and PARISxy irradiation largely dominate the intensity of the cross-peaks, leads itself to symmetric spectra where the integrated intensities of diagonal peaks are not necessarily proportional to the number of nuclei.

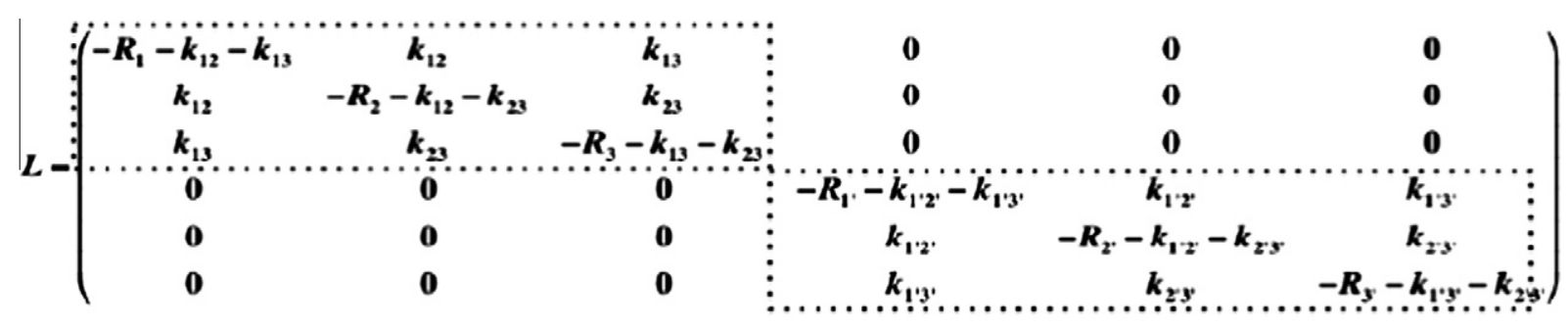

Table 1

Internuclear carbon-carbon distances $r_{\mathrm{ij}}$, zero-quantum relaxation times $\bar{T}_{2}^{(\mathrm{ZQ})}$, calculated rate constants $\overline{W_{i j}}$ and measured exchange rate constants $k_{\mathrm{ij}}$ in L-alanine.

\begin{tabular}{|c|c|c|c|c|c|c|}
\hline$i, j$ & $r_{i j}(\AA)$ & $\bar{T}_{2}^{(Z Q)}(\mathrm{s})$ & $\overline{W_{i j}}\left(\mathrm{~s}^{-1}\right)$ & $k_{i j}\left(\mathrm{~s}^{-1}\right)^{\mathrm{a}}$ & $k_{i j}\left(\mathrm{~s}^{-1}\right)^{\mathrm{b}} 1 \mathrm{st}$ population & $k_{i j}\left(\mathrm{~s}^{-1}\right)^{\mathrm{b}} 2$ nd population \\
\hline $\mathrm{CH}_{3}-\mathrm{CH}$ & 1.54 & $1.72 \pm 0.1 \times 10^{-5}$ & $29.2 \pm 1.7$ & $32.8 \pm 0.5$ & $15.1 \pm 0.05$ & $3.1 \pm 0.05$ \\
\hline $\mathrm{CH}-\mathrm{COOH}$ & 1.55 & $2.05 \pm 0.1 \times 10^{-5}$ & $33.5 \pm 1.8$ & $33.3 \pm 0.5$ & $22.8 \pm 0.05$ & $4.3 \pm 0.05$ \\
\hline $\mathrm{CH}_{3}-\mathrm{COOH}$ & 2.55 & $5.31 \pm 0.05 \times 10^{-5}$ & $4.4 \pm 0.6$ & $6.6 \pm 0.5$ & $1.1 \pm 0.05$ & $0.4 \pm 0.05$ \\
\hline
\end{tabular}

a Extracted by fitting experimental data using Eqs. (1), (2).

b By using the exchange matrix Eq. (7). 


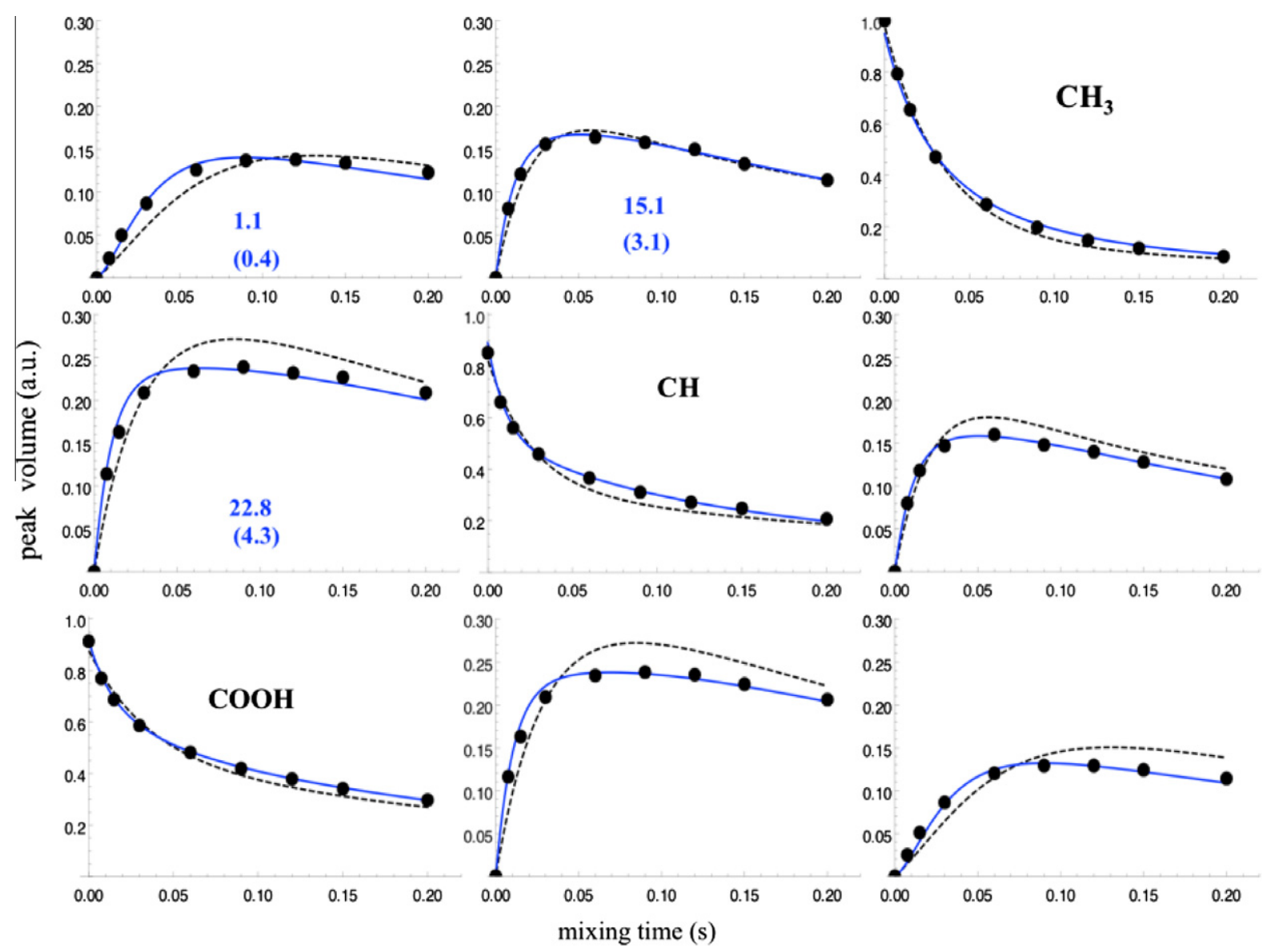

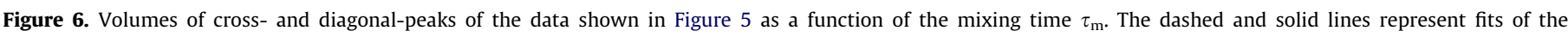

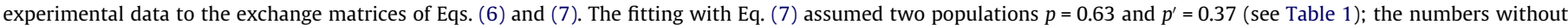
brackets give the exchange rates $\left( \pm 0.05 \mathrm{~s}^{-1}\right)$ for the first set $\{1,2,3\}$ while the numbers in brackets are those of the second set $\left\{1^{\prime}, 2^{\prime}, 3^{\prime}\right\}$.
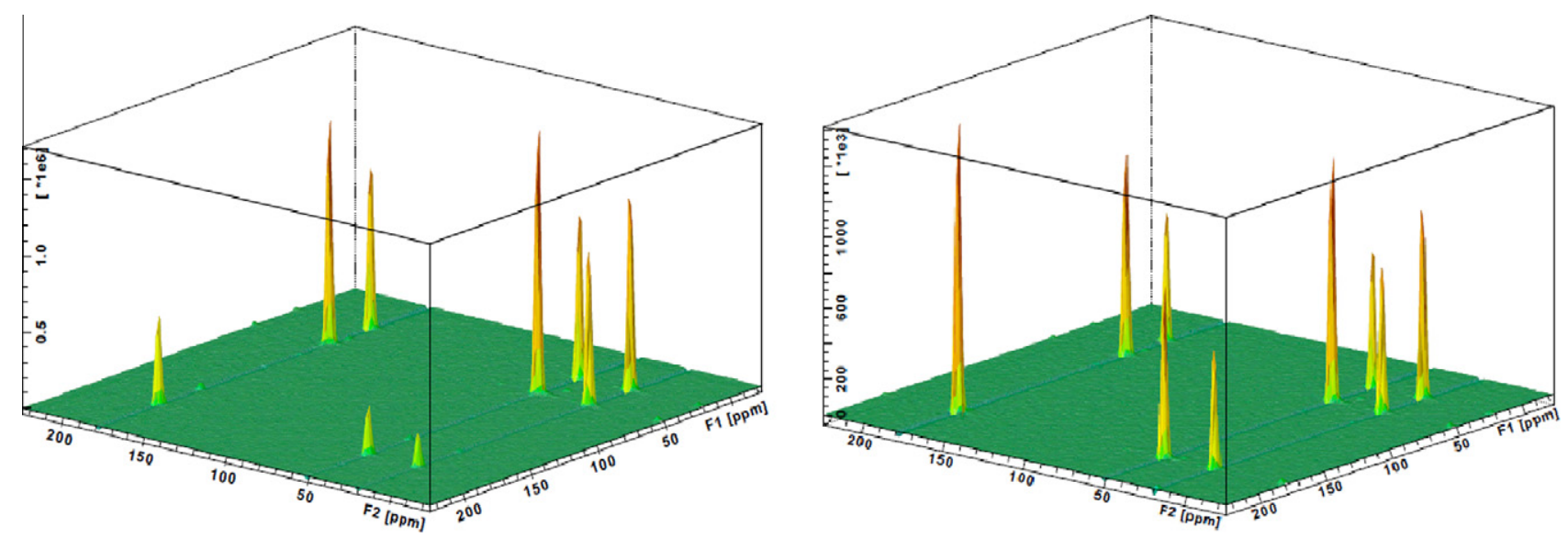

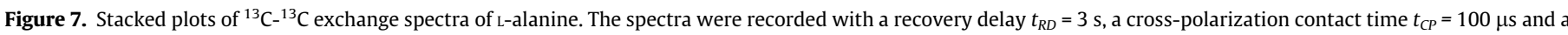

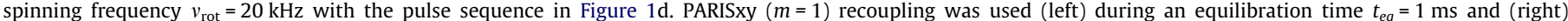

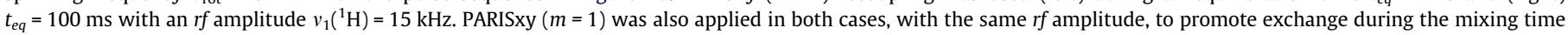
$\tau_{\mathrm{m}}=100 \mathrm{~ms}$.

To reach the latter requirement, the slowly exchanging populations should in principle come to equilibrium. Figure 8 shows the changes in the ${ }^{13} \mathrm{C}$ peak intensities of L-histidine as a function of the equilibration time $t_{e q}$ obtained using the pulse sequence of Figure 1 b with PARIS $(N=2)$ (top) and PARISxy $(m=1)$ (bottom). Under the present experimental conditions, both methods permit one to reach similar $( \pm 10 \%)$ magnetizations of chemically different nuclei on a time scale of a few hundreds of milliseconds, which compares favourably with other methods proposed to obtain quantitative CP-MAS spectra $[19,20]$. However, recording fully quantitative CP-MAS spectra would require a much longer equilibration time $t_{e q}$, depending on the amounts and rate constants of slowly exchanging populations. For given experimental conditions, this will depend on the choice of the methods used for equilibration. 


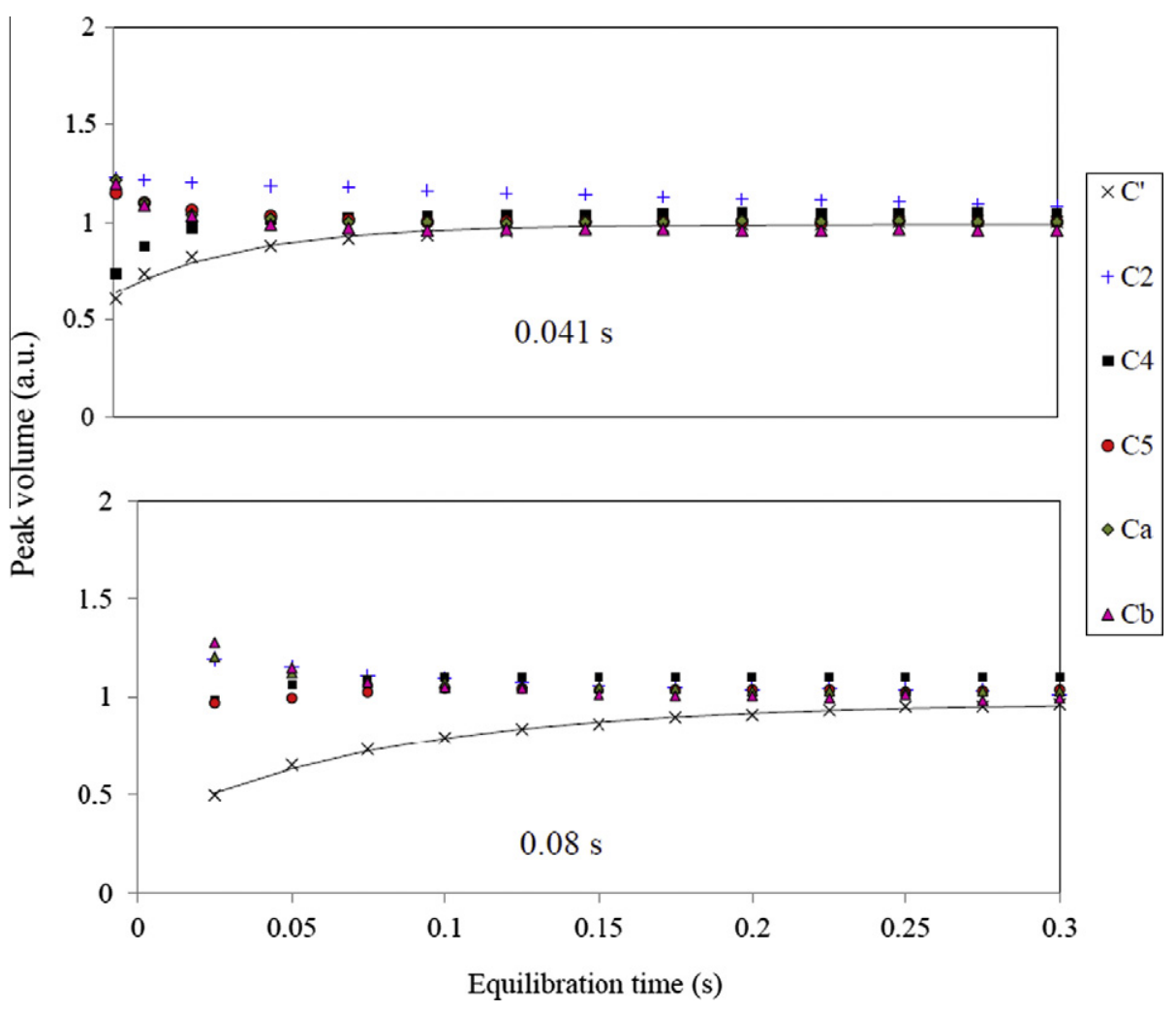

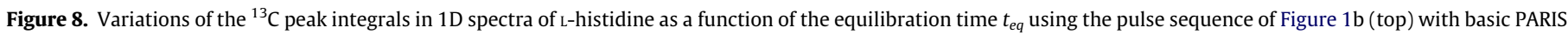

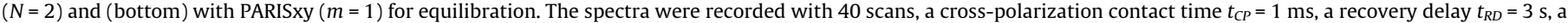

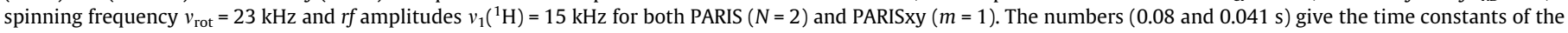
slow build-up of the $\mathrm{C}^{\prime}$ magnetization, obtained by exponential fitting (solid lines).

\section{Conclusions}

We have demonstrated that the intrinsic asymmetry of homonuclear solid-state NMR 2D correlation spectra arising from a non-uniform preparation of the magnetization can be removed by equilibration in the initial stage of exchange experiments by taking advantage of PARIS or PARISxy irradiation schemes. Both methods promote dipolar recoupling and permit an efficient magnetization exchange between spectrally close and distant carbons. This in turns allows one to create an equilibrated initial state, leading to the restoration of the symmetry about the main diagonal of 2D correlation spectra. Recording symmetric 2D spectra improves the quantitative analysis of exchange rates and helps the assignment of intra-residual and sequential cross-peaks, possibly assisted by covariance analysis [21], through the signal enhancement in 2D correlation spectra of biomolecules, microcrystalline proteins and amyloid fibrils. We have shown that both recoupling schemes, even when using modest $r f$ amplitudes, may also be exploited to record nearly quantitative 1D CP-MAS spectra.

\section{Acknowledgments}

Financial support from the Agence Nationale de la Recherche (Project 'FastSpinProts' ANR-09-BLAN-0111-01) and from the CNRS is gratefully acknowledged.

\section{References}

[1] R.R. Ernst, G. Bodenhausen, A. Wokaun, Principles of Nuclear Magnetic Resonances in One and Two Dimensions, Clarendon Press, Oxford., 1987.

[2] M. Weingarth, D. Demco, G. Bodenhausen, P. Tekely, Chem. Phys. Lett. 469 (2009) 342.

[3] M. Weingarth, G. Bodenhausen, P. Tekely, Chem. Phys. Lett. 488 (2010) 10.

[4] M. Weingarth, G. Bodenhausen, P. Tekely, J. Am. Chem. Soc. 131 (2009) 13937.

[5] M. Weingarth, Y. Masuda, K. Takegoshi, G. Bodenhausen, P. Tekely, J. Biomol. NMR 50 (2011) 129.

[6] J.E. Herbert-Pucheta, H. Colaux, G. Bodenhausen, P. Tekely, J. Phys. Chem. B 115 (2011) 15415.

[7] C.R. Morcombe, V. Gaponenko, R.A. Byrd, K. Zilm, J. Am. Chem. Soc. 126 (2004) 7196.

[8] S. Caldarelli, L. Emsley, J. Magn. Reson. 130 (1998) 233.

[9] M. Weingarth, P. Tekely, B. Bodenhausen, Chem. Phys. Lett. 466 (2008) 247.

[10] M. Weingarth, G. Bodenhausen, P. Tekely, J. Magn. Reson. 199 (2009) 238.

[11] M. Weingarth, G. Bodenhausen, P. Tekely, Chem. Phys. Lett. 502 (2011) 259.

[12] A. Abragam, The Principles of Nuclear Magnetism, Clarendon Press, Oxford, 1961.

[13] Z. Gan, R.R. Ernst, Chem. Phys. Lett. 253 (1996) 13.

[14] B.H. Meier, Adv. Magn. Opt. Reson. 18 (1994) 1.

[15] A. Kubo, C.A. McDowell, J. Chem. Soc. Faraday Trans.1 84 (1988) 3713.

[16] P. Tekely, M.J. Potrzebowski, Y. Dusausoy, Z. Luz, Chem. Phys. Lett. 291 (1998) 471.

[17] P. Tekely, C. Gardiennet, M.J. Potrzebowski, A. Sebald, D. Reichert, Z. Luz, J. Chem. Phys. 116 (2002) 7607.

[18] R. Ohashi, K. Takegoshi, J. Chem. Phys. 125 (2006) 214503.

[19] G. Hou, F. Deng, S. Ding, R. Fu, J. Yang, C. Ye, Chem. Phys. Lett. 421 (2006) 356.

[20] K. Takeda, Y. Noda, K. Takegoshi, O. Lafon, J. Trébosc, J.P. Amoureux, J. Magn. Reson. 214 (2012) 340.

[21] M. Weingarth, P. Tekely, R. Brüschweiler, G. Bodenhausen, Chem. Commun. 46 (2010) 952. 